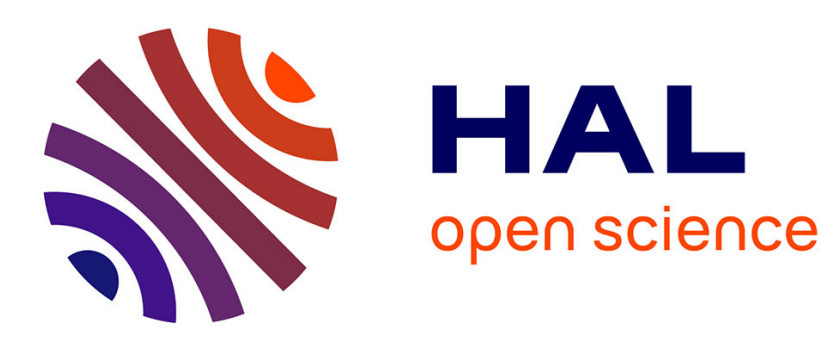

\title{
RÉSONANCE PARAMAGNÉTIQUE ÉLECTRONIQUE DES \\ NITROSYL-HÉMOPROTÉINES : APPLICATION A L'ÉTUDE DES INTERATIONS DANS L'HÉMOGLOBINE
}

Y. Henry

\section{To cite this version:}

Y. Henry. RÉSONANCE PARAMAgNÉTIQUE ÉLECTRONIQUE DES NITROSYLHÉMOPROTÉINES: APPLICATION A L'ÉTUDE DES INTERATIONS DANS L'HÉMOGLOBINE. Journal de Physique Colloques, 1973, 34 (C8), pp.C8-17-C8-20. 10.1051/jphyscol:1973810 . jpa-00215363

HAL Id: jpa-00215363 https://hal.science/jpa-00215363

Submitted on 1 Jan 1973

HAL is a multi-disciplinary open access archive for the deposit and dissemination of scientific research documents, whether they are published or not. The documents may come from teaching and research institutions in France or abroad, or from public or private research centers.
L'archive ouverte pluridisciplinaire HAL, est destinée au dépôt et à la diffusion de documents scientifiques de niveau recherche, publiés ou non, émanant des établissements d'enseignement et de recherche français ou étrangers, des laboratoires publics ou privés. 


\title{
RÉSONANCE PARAMAGNÉTIQUE ÉLECTRONIQUE DES NITROSYL-HÉMOPROTÉINES : APPLICATION A L'ÉTUDE DES INTERACTIONS DANS L'HÉMOGLOBINE
}

\author{
Y. HENRY
}

Institut de Biologie Physico-chimique, Paris

\begin{abstract}
Résumé. - Les spectres de résonance paramagnétique électronique des nitrosyl-hémoprotéines apportent des renseignements directs sur le complexe héminique ; l'oxyde azotique, ligand du fer de l'hème, apparaît comme un marqueur extrêmement sensible aux changements de conformation protéique. De plus l'analogie entre l'oxyde azotique et l'oxygène permet l'étude des mécanismes de réactions de très nombreuses hémoprotéines; en particulier, l'application à l'étude des interactions dans l'hémoglobine a permis de caractériser le degré d'inéquivalence structurale et fonctionnelle des sous-unités protéiques et de suggérer fortement l'existence d'intermédiaires conformationnels dans les réactions de I'hémoglobine.
\end{abstract}

\begin{abstract}
Electron paramagnetic resonance spectra of nitrosylhemoproteins bring direct information on the heme complex; nitric oxide has been found to be an extremely sensitive probe for protein conformational changes. Furthermore using the analogy between oxygen and nitric oxide the study of reactional mechanisms of many hemoproteins is possible; for example, in the study of the heme-heme interaction in hemoglobin we have characterized the degree of structural and functional unequivalence between the two types of protein subunits and suggested strongly the existence of conformational intermediates in hemoglobin reactions.
\end{abstract}

Les sondes paramagnétiques. - La connaissance de la nature de l'environnement protéique du groupement prosthétique est essentielle pour comprendre Ia diversité des fonctions remplies par les hémoprotéines : transport d'oxygène, réduction des peroxydes, transfert d'électrons. Beaucoup d'entre elles ont le même groupe prosthétique, le protohène IX ; c'est le cas de l'hémoglobine, de la myoglobine, des peroxydases, des catalases, des cytochromes b. Pour d'autres hémoprotéines le groupe prosthétique, bien que différent de l'hème IX, est également un dérivé porphyrinique : par exemple, l'hème a pour la cytochrome-c-oxydase (cytochrome $\mathrm{aa}_{3}$ ), l'hème $\mathrm{c}$ pour le cytochrome $c$.

Pour certaines de ces protéines, hémoglobine, myoglobine, cytochrome $\mathrm{c}$, un examen détaillé de la structure de l'hème et de son enviromnement est possible par la cristallographie aux rayons $X$; en particulier, le ou les ligands protéiques et le ligand exogène éventuel du fer sont connus de manière non équivoque. Dans le cas des peroxydases et de la plupart des cytochromes, malgré l'abondance de données récentes, les informations structurales précises restent encore très limitées.

L'usage des sondes spectroscopiques et paramagnétiques a donné de nombreuses informations sur les changements de conformations qui se produisent sur la protéine au voisinage plus ou moins immédiat de l'hème. Les marqueurs de spin électronique ou nucléaire peuvent être attachés à certains résidus de l'apoprotéine (Ogawa et McConnell [20], Huestis et Raftery [15]) et à l'hème lui-même sur certains groupements de la porphyrine (Asakura et al. [2]). On a aussi utilisé un marqueur de spin portant le groupe isocyanure comme ligand exogène du fer de l'hème du cytochrome P-450 (Reichman et al. [21]). L'inconvénient fondamental de ces marquages réside dans lampleur de la perturbation apportée par le marqueur et dans la difficulté à estimer l'importance de cette perturbation (Moffat [19]). D'autre part les interprétations structurales qu'on peut tirer des modifications des résonances du marqueur restent en général ambiguës.

Le complexe héminique lui-même, c'est-à-dire l'hème et le ou les deux ligands axiaux du fer, peut être considéré dans l’étude de ces proténes comme un marqueur: le problème des perturbations ne se pose plus puisque celles-ci s'identifient aux modifications mêmes que l'on cherche ì caractériser. Les hémoprotéines ferriques en particulier sont paramagnétiques et l'étude des spectres d'absorption optique, la mesure des susceptibilités magnétiques et l'étude des spectres de résonance paramagnétique électronique (RPE) permettent souvent de définir l'état de spin du fer dans le complexe et certains paramètres de la symétrie du champ des ligands. On peut suivre par de telles études les modifications de structure électronique du complexe héminique liées à des modifications de structure tridimensionnelle de l'apoprotéine. 
Résonance paramagnétique électronique des nitrosylhémoprotéines. - Nous nous intéresserons ici à une sonde paramagnétique de l'hème, qui a été assez peu utilisée jusqu'à présent et dont l'usage présente un intérêt général pour l'étude des hémoprotéines: il s'agit de l'oxyde azotique. Cette molécule paramagnétique est un ligand $\mathrm{du}$ fer des hèmes ferreux et ferriques. Le complexe nitrosylé des hémoprotéines réduites contient encore un électron célibataire: l'oxyde azotique joue donc le rôle de marqueur de spin et de ligand. Comme dans le cas des hémoprotéines ferriques de spin fort et de spin faible, l'oxyde azotique lié à une hémoprotéine ferreuse a l'avantage très important d'être une sonde directe du complexe héminique, le site de réaction, à l'encontre des marqueurs de spin classique. Les spectres de RPE de plusieurs nitrosyl-hémoprotéines ont été récemment étudiés : l'hémoglobine et ses sous-unités (Kon [16], Chien [7], Shiga et al. [22], Henry et Banerjee [13]), la myoglobine (Dickinson et Chien [10]), le cytochrome $\mathbf{c}$ (Kon [18]), la peroxydase de raiefort, la cytochrome-c-oxydase et la catalase (Yonetani et al. [25]) et divers cytochromes (Cox et al. [8], Blokzijl-Homan et Van Gelder [6], Dervartanian et Legall [9]). Dans de nombreux cas on a pu obtenir des renseignements structuraux précis en s'aidant des interprétations dérivées par Kon et Kataoka [17] de leur étude de complexes héminiques modèles.

Un spectre de résonance paramagnétique électronique de la nitrosyl-hémoglobine fut pour la première fois enregistré par Bennett et al. [5]. Plus tard Kon [16] et Kon et Kataoka [17] montrèrent que le centre paramagnétique a une symétrie rhombique et que l'électron célibataire de l'oxyde azotique est fortement délocalisé sur le fer de l'hème et sur l'azote de l'histidine dite proximale, le ligand protéique de l'hème. L'orbitale est du type $\mathrm{dz}^{2}-\sigma^{*}$. Il y a de plus une forte interaction $\mathrm{d} \pi-\mathrm{p}_{\pi}$ des électrons $\pi$ de la base et $d \pi\left(d_{X Z}, d_{Y Z}\right)$ du fer. Ils montrèrent de plus que le spectre est extrêmement sensible aux changements de la conformation de l'hémoglobine.

Bien que les structures électroniques de $\mathrm{NO}$ et de $\mathrm{O}_{2}$ soient différentes elles présentent certaines analogies (Harcourt [12]) qu'on retrouve dans les formules qu'on peut écrire pour $\mathrm{HbNO}$ et $\mathrm{HbO}_{2}$. La délocalisation de l'électron célibataire montrée pour $\mathrm{HbNO}$ rappelle d'ailleurs la suggestion faite par Weiss que $\mathrm{HbO}_{2}$ est un complexe contenant l'ion superoxyde $\mathrm{Fe}-\mathrm{OO}^{-}$(Weiss [23]). Cette suggestion a tout récemment reçu un appui expérimental (Wever et al. [24]).

Shiga et al. [22], puis Henry et Banerjee [13] ont ensuite montré que le spectre de RPE de HbNO est en fait la somme des spectres propres aux deux types de chaînes $\alpha$ et $\beta$ qui composent le tétramère. Les spectres des sous-unités isolées $\alpha$ NO et $\beta$ NO sont radicalement différents : alors que le spectre de $\alpha$ NO dénote pour le centre paramagnétique une symétrie nettement rhombique, celle-ci pour $\beta \mathrm{NO}$ apparaît quasi axiale. De plus le mode d'interaction super-hyperfine apparaît totalement différent dans les deux protéines : la présence de neuf lignes régulièrement espacées, se groupant en un triplet de triplets, s'interprète pour $\alpha$ NO par l'interaction avec le noyau ${ }^{14} \mathrm{~N}$ de $\mathrm{NO}$ (19 gauss d'écart) et avec le noyau ${ }^{14} \mathrm{~N}_{\varepsilon}$ de l'imidazole de l'histidine proximale (6,5 gauss d'écart). Au contraire pour $\beta \mathrm{NO}$ aucune structure super-hyperfine n'est clairement visible. La comparaison de quatre nitrosyl-hémoprotéines aussi semblables que $\alpha \mathrm{NO}, \beta \mathrm{NO}, \gamma \mathrm{NO}$ (dérivé de l'hémoglobine fretale humaine $\alpha_{2} \gamma_{2}$ ) et MbNO, qui possèdent toutes le même ligand protéique $\mathrm{His}(\mathrm{F} 8)$ et une histidine « distale » His (E 7), et qui ont, à la résolution de $5,5 \AA$, des structures tertiaires semblables, montre que les spectres de RPE sont très variés. $\gamma \mathrm{NO}$ et MbNO ont des spectres intermédiaires entre ceux de $\alpha \mathrm{NO}$ et de $\beta \mathrm{NO}$ à la fois quant à la symétrie et quant à l'interaction superhyperfine. On peut supposer que ces différences spectrales prononcées correspondent à des différences de structures tertiaires assez faibles.

La comparaison avec les spectres des autres nitrosyl-hémoprotéines étudiées montre encore plus de diversité. A la suite du travail de Kon et Kataoka [17] sur des complexes modèles composés du nitrosyl-hème et d'une base azotée on peut attribuer les résonances en se référant aux axes orthogonaux, OX et $O Y$ dans le plan de l'hème et $\mathrm{OZ}$ suivant sa perpendiculaire. Pour les hémoprotéines étudiées $g_{X}$ varie en général assez peu de 2,06 à 2,10, de même que $g_{Z}$ de 2,001 à 2,005 ; au contraire $g_{\mathrm{Y}}$ peut varier entre 1,95 et 2,05 . Ainsi la symétrie du complexe paramagnétique apparaît très variable en particulier par la distorsion rhombique. En général l'absorption correspondant à $g_{z}$ présente une structure superhyperfine plus ou moins résolue. Celle-ci peut être composée de neuf raies, qu'on peut grouper en un triplet de triplets. Le triplet, de 16 à 24 gauss d'écart est dû à l'interaction avec le noyau ${ }^{14} \mathrm{~N}(I=1)$ de NO; le triplet, de 6,4 à 6,8 gauss d'écart peut être attribué à l'azote de la base, ligand du fer. Ceci permet parfois de faire des hypothèses sur le ligand protéique de l'hème pour les hémoprotéines où il n'est pas connu : ainsi on a suggéré que ce ligand est un résidu d'histidine dans le cas de la peroxydase de raiefort [25] et dans celui du cytochrome $\mathrm{aa}_{3}$ [6]. Dans certains cas le triplet de triplets coalesce en un seul triplet dû à NO. La variation de l'écart de la structure superhyperfine due à NO s'interprète par des orientations très diverses de NO par rapport à l'hème.

Un autre intérêt de l'étude de RPE de ces systèmes est de pouvoir, dans certains cas, suivre une voie métabolique et de caractériser les enzymes responsables : ainsi Cox et al. [8] ont pu montrer que des cytochromes de type $\mathrm{c}$ interviennent directement dans la réduction du nitrite en oxyde azotique et dans celle de l'oxyde azotique NO en oxyde azoteux $\mathrm{N}_{2} \mathrm{O}$. Des signaux de RPE caractéristiques de nitrosyl- 
hémoprotéines apparaissent dans certains tissus tumoraux (Emanuel et al. [11]); bien que les protéines ne soient pas isolées ni caractérisées et qu'on ne connaisse pas les réactions en jeu, de tels signaux sont très utiles pour la détection de ces tumeurs.

Application à l'étude des interactions dans l'hémoglobine. - C'est dans l'étude de la nitrosyl-hémoglobine et des interactions entre ses sous-unités que l'usage de l'oxyde azotique comme sonde paramagnétique a été le plus développé. Ceci est dû à l'analogie fonctionnelle directe entre l'oxyde azotique et l'oxygène dans leur équilibre avec l'hémoglobine: la fixation de NO à la déoxyhémoglobine a en effet le même caractère coopératif que celle de l'oxygène. On peut donc penser que ces deux réactions font intervenir le même phénomène d'interaction entre les hèmes suivant le même processus conformationnel.

La cristallographie aux rayons $X$ a permis de définir les structures tertiaires, au niveau de chaque sous-unité $\alpha$ et $\beta$, et quaternaires, au niveau de la molécule tétramérique $\alpha_{2} \beta_{2}$, de la déoxyhémoglobine et de l'oxyhémoglobine. Le phénomène d'interaction est alors décrit en termes stéréochimiques par la transition entre les conformations quaternaires de la molécule déoxygénée ( $T$, tendue) et de la molécule oxygénée ( $R$, relâchée). Jusqu'à présent cette méthode ne donne aucun renseignement direct sur les propriétés des intermédiaires éventuels de la réaction d'oxygénation; de plus les relations entre l'état de spin du fer de l'hème et la conformation protéique et le degré d'inéquivalence structurale des sous-unités restent encore imprécises. La possibilité de préparer les molécules tétramériques à caractère hybride telles que $(\alpha \mathrm{NO})_{2} \beta_{2}$ et $\alpha_{2}(\beta \mathrm{NO})_{2}$ (Antonini et al. [1], Henry et Banerjee [13]) ou $\alpha_{2}^{\text {III }} \beta_{2}$ et $\alpha_{2} \beta_{2}^{\text {III }}$ (Banerjee et Cassoly [3]) a ouvert des perspectives nouvelles d'expérimentations. Du fait des analogies structurales entre l'oxyhémoglobine et la ferrihémoglobine ou la nitrosylhémoglobine, les molécules hybrides stables sont a priori semblables aux intermédiaires fugaces qui peuvent se produire lors de l'oxygénation. Ces molécules ont d'autre part une grande utilité opérationnelle pour l'étude des interactions homotropes, car on peut dans de tels tétramères mettre en branle une séquence d'événements en modifiant un hème et en observant un autre hème de manière spécifique et sélective (Banerjee et al. [4]).

L'étude par RPE des complexes nitrosylés des hybrides $(\alpha \mathrm{NO})_{2} \beta_{2}$ a montré (Henry et Banerjee [13]) que le changement d'état de spin des chaînes $\beta$ induit un changement dans la structure électronique des chaînes $\alpha \mathrm{NO}$ qui leur sont associées : en effet les hybrides $(\alpha \mathrm{NO})_{2} \quad\left(\beta \mathrm{O}_{2}\right)_{2}, \quad(\alpha \mathrm{NO})_{2}(\beta \mathrm{CO})_{2}$, $(\alpha \mathrm{NO})_{2}\left(\beta^{\text {III }} \mathrm{CN}^{-}\right)_{2}$ où les hèmes $\beta$ sont de spin nul $(S=0)$ ou faible $(S=1 / 2)$ ont des spectres de RPE identiques à celui de la chaîne $\alpha$ NO isolée; par contre les hybrides $(\alpha \mathrm{NO})_{2}(\beta \text { deoxy })_{2}$, $(\alpha \mathrm{NO})_{2}\left(\beta^{1 \mathrm{II}} \mathrm{F}^{-}\right)_{2}, \quad(\alpha \mathrm{NO})_{2} \quad\left(\beta^{\mathrm{III}} \mathrm{H}_{2} \mathrm{O}\right)_{2}$ où les hèmes $\beta$ sont de spin fort $(S=2$ ou $S=5 / 2)$ ont des spectres modifiés. Ces modifications sinterprètent essentiellement par une altération de l'orbitale $\mathrm{d} z^{2}-\sigma^{*}$ qui participe aux liaisons du fer avec ses deux ligands axiaux par rapport à l'hème. La propagation d'une information dans le sens inverse $\beta \rightarrow x$ n'a pu être mise en évidence dans les hybrides de type $\alpha_{2}(\beta N O)_{2}$. Les données de cristallographie ont conduit à mettre l'accent sur le rôle joué par l'état de ligation dans le mécanisme d'interaction entre les sous-unités: elles distinguent en effet la structure quaternaire "deoxy » (Fe ${ }^{I I}$ penta coordiné) de la structure quaternaire " oxy » (hémoglobine ferreuse ou ferrique « liée »). Nos expériences mettent au contraire en relief le rôle dans le phénomène d'interaction du changement d'état de spin des hèmes et du changement de structure tertiaire concomittant. Cette étude des hybrides a permis aussi de généraliser le caractère d'inéquivalence structurale des sousunités, isolées ou dans un tétramère.

Grâce à ces données structurales nous avons pu étudier par RPE les cinétiques d'association et de dissociation de NO à l'hémoglobine. On a pu montrer (Henry et Cassoly [14]) que lors de la combinaison il y a une association nettement préférentielle sur les chaines $\alpha$. On a pu estimer que la valeur du rapport des constantes cinétiques d'association pour le premier site $(\alpha$ ou $\beta) j_{1 x}^{\prime} / j_{1 \beta}^{\prime}$ est voisin de six. Il peut même y avoir une compétition entre le second site $\alpha$ et le premier site $\beta$. Dans la réaction de dissociation le ligand NO se dissocie environ deux fois plus vite de la chaîne $\beta$ que de la chaîne $\alpha$, c'est-à-dire $j_{4 \alpha} / j_{4 \beta}=2$. Ces expériences montrent qu'à l'inéquivalence structurale des deux types de chaines correspond une inéquivalence fonctionnelle et que les intermédiaires fonctionnels naturels existent. En particulier, des molécules telles que $\alpha \mathrm{NO} \alpha \beta \beta$ et $\alpha \mathrm{NO} \alpha \mathrm{NO} \beta \beta$ ont une durée de vie non négligeable, ce qui justifie a posteriori l'étude des hybrides artificiels $(\alpha \mathrm{NO})_{2} \beta_{2}$.

Les études que nous avons menées sur l'hémoglobine par RPE en nous fondant sur l'analogie entre NO et $\mathrm{O}_{2}$ nous permettent donc de suggérer lexistence des intermédiaires conformationnels et de caractériser l’inéquivalence structurale et fonctionnelle des sous-unités. Bien que les études sur les autres hémoprotéines aient été encore peu développées, elles permettent de souligner les avantages généraux de l'oxyde azotique comme sonde paramagnétique. La perturbation qu'elle apporte est bien définie puisqu'il s'agit d'un ligand du fer de l'hème; de plus la variété des spectres de RPE obtenus et de leurs modifications possibles montre que cette sonde est particulièrement sensible aux différences et aux altérations de la structure protéique qui se répercutent au niveau de l'hème lui-même; la sensibilité de cette sonde est en particulier beaucoup plus grande que celle des complexes ferriques de spin fort ou faible. Enfin, sachant que l'oxygène forme des complexes, 
parfois hypothétiques du fait de leur fugacité, avec de nombreuses hémoprotéines ferreuses au cours de leur réaction de catalyse, l'analogie entre l'oxygène et l'oxyde azotique rend l'utilisation de ce dernier particulièrement intéressante pour l'étude des mécanismes de réaction.

\section{Bibliographie}

[1] Antonini, E., Brunori, M., Wyman, J. et Noble, R. W., J. Biol. Chem, 241 (1966) 3236.

[2] Asakura, T., Leigh, J. S., Drott, H. R., Yonetani, T. et Chance, B., Proc. Nat. Acad. Sci. USA 68 (1971) 861.

[3] Banerjee, R. et Cassoly, R., J. Mol. Biol. 42 (1969) 351.

[4] Banerjee, R., Stetzkowski, F. et Henry, Y., J. Mol. Biol. 73 (1973) 455.

[5] Bennett, J. E., Ingram, D. J. E., George, P. et Griffith, J. S., Nature 176 (1955) 394.

[6] BlokziJl-Homan, M. F. J. et Van Gelder, B. F., Biochim. Biophys. Acta 234 (1971) 493.

[7] Chien, J. C. W., J. Chem. Plys. 51 (1969) 4220.

[8] Cox, C. D., Payne, W. J. et Dervartanian, D. V., Biochim. Biophys. Acta 253 (1971) 290.

[9] DerVartanian, D. V. et Legall, J., Biochim. Biophys. Acta 243 (1971) 53.

[10] Dickinson, L. C. et Chien, J. C. W., J. Amer. Chem. Soc. 93 (1971) 5036.

[11] Emanuel, N. M., Saprin, A. N., Shabalkin, V. A., Kozlova, L. E. et Krugluakova, K. E., Nature 222 (1969) 165.
[12] Harcourt, R. D., Biopolymers 11 (1972) 1551.

[13] Henry, Y. et Banerjee, R., J. Mol. Biol. 73 (1973) 469.

[14] Henry, Y. et Cassoly, R., Biochem. Biophys. Res. Comm. 51 (1973) 659.

[15] Huestis, W. H. et Raftery, M. A., Proc. Nat. Acad. Sci. USA 69 (1972) 1887.

[16] Kon, H., J. Biol. Chem. 243 (1968) 4350.

[17] Kon, H. et KataOKa, N., Biochemistry 8 (1968) 4757.

[18] Kon, H., Biochem. Biophys. Res. Comm. 35 (1969) 423.

[19] Moffat, J. K., J. Mol. Biol, 55 (1971) 135.

[20] Ogawa, S. et McConnell, H. M., Proc. Nat. Acad. Sci. USA 58 (1967) 19.

[21] Reichman, L. M., Annaey, B., Belova, V. S. et Rozantzev, E. G., Nature New Biol. 237 (1972) 31.

[22] Shiga, T., Hwang, K. J. et Tyuma, I., Biochemistry 8 (1969) 378.

[23] WeIss, J. J., Nature 202 (1964) 83 et 202 (1964) 183.

[24] Wever, R., Ondega, B. et Van Gelder, B. F., Biochim Biophys. Acta 302 (1973) 475.

[25] Yonetani, T., Yamamoto, H., Erman, J. E., Leigh, J. S. et Reed, G. H., J. Biol. Chem. 247 (1972) 2447. 